\title{
Von der Validierung zur Übersetzung. Passungsprobleme zwischen formaler und institutioneller Anerkennung in (erwachsenen)pädagogischen Kontexten
}

\author{
Nico Sturm (1D)
}

Eingegangen: 7. Juni 2018 / Angenommen: 9. Oktober 2018 / Online publiziert: 19. Oktober 2018 (C) Der/die Autor(en) 2018

Zusammenfassung In den vergangenen 15 Jahren sind in (erwachsenen-)pädagogischen Kontexten vielfältige Verfahren entwickelt worden, um formal, non-formal und informell erworbene Kompetenzen zu validieren und somit im Sinne des Lebenslangen Lernens nutzbar machen zu können. Trotz der Entwicklung, Erprobung und Evaluation entsprechender Verfahren sowie prozessbegleitender Forschungsarbeiten und prozeduraler Klärungsprozesse, ist eine flächendeckende Implementierung nicht zu beobachten. Aus diesen Erkenntnissen kann die Annahme abgeleitet werden, dass sich der geringe Umsetzungsgrad entsprechender Verfahren durch Passungsprobleme zwischen der formalen Anerkennung und der institutionellen Anerkennung begründen lässt. Somit bestünde die zentrale Problematik nicht primär in der Validierung von Kompetenzen anhand praktikabler, valider und reliabler Verfahren, sondern in der Übersetzung der validierten Kompetenzen in die jeweiligen institutionellen Kontexte der anerkennenden Institutionen. Diese These wird am Beispiel des Feldes der wissenschaftlichen Weiterbildung exemplarisch überprüft, um die Erkenntnisse abschließend zu generalisieren und für andere Institutionen der Erwachsenenbildung nutzbar zu machen.

Schlüsselwörter Anrechnung - Anerkennung · Non-formale Kompetenzen · Informelle Kompetenzen · Übersetzungsprobleme · Begriffserweiterung

Publisher's Note Springer Nature remains neutral with regard to jurisdictional claims in published maps and institutional affiliations.

Dr. N. Sturm $(\bowtie)$

Philipps-Universität Marburg, Marburg, Deutschland

E-Mail: nico.sturm@uni-marburg.de 


\title{
From validation to translation. Matching problems between formal and institutional recognition in (adult) education contexts
}

\begin{abstract}
Over the past 15 years, a variety of approaches have been developed in (adult) education contexts to validate formal, non-formal and informal competences and thus harness them for lifelong learning purposes. Despite the development, testing and evaluation of appropriate procedures as well as process-accompanying research and procedural clarification processes, a nationwide implementation can not be observed. It can be deduced from these findings that the low degree of implementation of corresponding procedures can be explained by matching problems between formal recognition and institutional recognition. Thus, the central problem would not be primarily the validation of competences using workable, valid and reliable procedures, but the translation of validated competences into the respective institutional contexts of the recognizing institutions. Using the example of the field of continuing education, this thesis will be examined as an example in order to generalize the findings and make them usable for other adult education institutions.
\end{abstract}

Keywords Recognition $\cdot$ Crediting $\cdot$ Non-formal competences $\cdot$ Informal competences $\cdot$ Translation issues $\cdot$ Term extension

\section{Einleitung}

In den vergangenen 15 Jahren gab es vielfältige Bemühungen non-formal und informell erworbene Kompetenzen $\mathrm{zu}$ ermittel ${ }^{1}$ und $\mathrm{zu}$ validieren ${ }^{2}$, um sie somit im Sinne des lebenslangen Lernens für unterschiedliche (erwachsenen-)pädagogische Kontexte nutzbar machen zu können. Im Bereich der Berufsbildung gab es sowohl auf europäischer Ebene (vgl. Europäisches Zentrum für die Förderung der Berufsbildung (CEDEFOP) 2009, 2016) als auch auf nationaler Ebene (Federal Institute for Vocational Education and Training 2016) bereits intensive Bemühungen, Rahmenbedingungen und Leitlinien für die Validierung von non-formal und informell erworbenen Kompetenzen zu schaffen. Entsprechende Maßnahmen im Bereich der hochschulischen Bildung wurden auf europäischer Ebene mit dem Memorandum über Lebenslanges Lernen (Europäische Kommission 2000) proklamiert, welches die ,gegenseitige Anerkennung“ zwischen den verschiedenen Lernbereichen (formal, non-formal, informell) betont (vgl. ebd., S. 12). Das Londoner Kommuniqué aus dem Jahr 2007 nimmt eine weitere Konkretisierung vor, indem es die Anerken-

\footnotetext{
1 Die Ermittlung von non-formalem und informellem Lernen wird als ein Prozess verstanden, der die Lernergebnisse einer Einzelperson festhält und sie erkennbar macht. Sie führt nicht zur Ausstellung eines offiziellen Zeugnisses oder Diploms, kann aber die Grundlage für eine offizielle Anerkennung schaffen (Europäisches Zentrum für die Förderung der Berufsbildung (Cedefop) S. 17).

2 Die Validierung von Lernergebnissen meint die Bestätigung durch eine zuständige Behörde oder Stelle, dass Lernergebnisse (Kenntnisse, Fertigkeiten und/oder Kompetenzen), die eine Person in einem formalen, nicht formalen oder informellen Kontext erzielt hat, gemäß festgelegten Kriterien bewertet wurden und den Anforderungen eines Validierungsstandards entsprechen. Die Validierung führt üblicherweise zur Zertifizierung (Europäisches Zentrum für die Förderung der Berufsbildung (Cedefop) S. 89).
} 
nung von Vorkenntnissen einschließlich der Bereiche des nicht-formellen und informellen Lernens fordert (vgl. Londoner Kommuniqué 2007, S. 3). Auf nationaler Ebene beschloss die Kultusministerkonferenz (KMK) im Jahr 2002 eine Anrechnung von außerhalb des Hochschulraumes erworbenen Kompetenzen grundsätzlich zu ermöglichen, insofern eine Gleichwertigkeit der anzurechnenden Vorleistungen mit den Studieninhalten festgestellt werden kann (vgl. KMK 2002). Weitere Beschlüsse zu Fragen der Gleichwertigkeit, (vgl. KMK 2008) sowie die ländergemeinsamen Strukturvorgaben (vgl. KMK 2010, 2011) bilden die Grundlage für die gesetzlichen Regelungen in den jeweiligen Landeshochschulgesetzen.

Diese bildungsbereichsübergreifenden Aktivitäten zur Schaffung von Rahmenbedingungen und Leitlinien wurden und werden durch umfangreiche Förderprogramme sowohl auf dem Feld der Berufsbildung (Abschlussbezogene Validierung non-formal und informell erworbener Kompetenzen (ValiKom); Prototyping Transfer - Berufsanerkennung mit Qualifikationsanalysen) als auch auf dem Feld der hochschulischen Bildung (ANKOM-Initiative; Bund-Länder Wettbewerb „Aufstieg durch Bildung“: offene Hochschulen) flankiert. Ein wesentliches Ergebnis dieser Förderprogramme sind vielfältige Verfahren zur Ermittlung und Validierung non-formal und informell erworbener Kompetenzen. ${ }^{3}$

Diese vielfältigen Bemühungen haben jedoch nicht $\mathrm{zu}$ einer breiten Zunahme wechselseitiger Durchlässigkeit zwischen beruflicher und akademischer Bildung geführt. Für den Bereich der akademischen Bildung muss festgestellt werden, dass sich deutsche Hochschulen der Anrechnungsthematik nur zögerlich annähern und darüber hinaus im internationalen Vergleich zurückhängen (vgl. Voigt 2012, S. 171). Auch andere empirische Erhebungen (vgl. Faulstich et al. 2007; Hanak und Sturm 2015a) kommen zu dem Schluss, dass eine Implementierung von qualitätsgesicherten und transparenten Anrechnungsverfahren in der Breite der Hochschullandschaft bisher nicht hinreichend stattgefunden hat. So kommen Verfahren zur Erhöhung der wechselseitigen Durchlässigkeit zwischen beruflicher und hochschulischer Bildung nicht über einzelne (meist drittmittelfinanzierte) Modellprojekte (vgl. exemplarisch das AAEK-Verfahren der Frankfurt University of Applied Sciences ${ }^{4}$ ) hinaus.

Im Rahmen des vorliegenden Beitrages wird daher der Frage nachgegangen, ob sich der geringe Umsetzungsgrad, anstatt mit einem Mangel an praktikablen, validen und reliablen Verfahren, mit Passungsproblemen zwischen der formalen Anerkennung (durch die vorliegenden Verfahren) und der institutionellen Anerkennung (durch die jeweilige (erwachsenen-)pädagogische Institution) erklären lässt. Dazu erfolgt eine exemplarische Betrachtung anhand des Feldes der wissenschaftlichen Weiterbildung.

Diese bietet sich insbesondere deshalb an, da - katalysiert durch umfassende Förderprogramme - im Bereich der wissenschaftlichen Weiterbildung intensive Bemühungen unternommen wurden, außerhochschulisch erworbene Kompetenzen auf ihre Gleichwertigkeit zu akademisch vermittelten Kompetenzen zu überprüfen. In den letzten Jahren sind vielfältige pauschale, individuelle und kombinierte Verfah-

\footnotetext{
3 Für einen breiten Überblick über Verfahren zur Kompetenzmessung siehe Erpenbeck et al. (2017).

4 Für weiterführende Informationen siehe https://www.frankfurtuniversity.de/de/hochschule/fachbereich4-soziale-arbeit-gesundheit/anrechnungsverfahren/.
} 
ren zur Anerkennung und Anrechnung formal, non-formal und informell erworbener Kompetenzen (siehe hierzu exemplarisch Müskens et al. 2013; Luft et al. 2018) für hochschulische Studienprogramme entstanden. Zudem ermöglichte die Drittmittelförderung eine Vielzahl prozessbegleitender empirischer Studien (siehe hierzu exemplarisch Damm 2018; Freitag et al. 2015; Hanak und Sturm 2015a,b; Schäfer et al. 2015; Seger et al. 2017).

Durch Rückgriff auf sowie eine systematische (Re-)Analyse von ausgewählten Forschungsergebnissen sollen mögliche Passungsprobleme zwischen formaler und institutioneller Anerkennung identifiziert, generalisiert und so für weitere (erwachsenen-)pädagogische Kontexte nutzbar gemacht werden. Gleichzeitig werden diese Betrachtungen genutzt, um die Begriffsarchitektur des Feldes einer kritischen Reflexion zu unterziehen.

Vor diesem Hintergrund werden in einem ersten Schritt die für das Feld zentralen Begrifflichkeiten definiert (Abschn. 2). In einem zweiten Schritt erfolgt eine Analyse möglicher Passungsprobleme zwischen formaler und institutioneller Anerkennung am Beispiel des Feldes der wissenschaftlichen Weiterbildung anhand einer Segmentierung in drei Ebenen (Abschn. 3). Aus einer kombinatorischen Zusammenschau der Analyseergebnisse erfolgt in einem dritten Schritt in Form einer Ergebnisdarstellung sowohl eine Rückbindung der Erkenntnisse auf die Frage der Passungsprobleme zwischen formaler und institutioneller Anerkennung als auch eine Bewertung möglicher Leerstellen in der Begriffsarchitektur des Feldes (Abschn. 4). In einem vierten und letzten Schritt werden die, am Beispiel der wissenschaftlichen Weiterbildung generierten, Ergebnisse generalisiert und hinsichtlich ihres Adaptierungspotentials auf die berufliche Bildung im Speziellen und andere (erwachsenen-)pädagogische Kontexte im Allgemeinen hin bewertet (Abschn. 5).

\section{Begriffsdefinitionen}

Um ein einheitliches Verständnis sicherzustellen, werden im Folgenden die für den vorliegenden Beitrag zentralen Begrifflichkeiten erläutert. Dies ist aus unterschiedlichen Perspektiven heraus notwendig. Während im Kontext der Berufsbildung die Begrifflichkeiten der Ermittlung (erkennbar machen von Lernergebnissen), der Validierung (Bestätigung durch die zuständige Behörde) und der Zertifizierung (Prozess zur Ausstellung eines Befähigungsnachweises) ${ }^{5}$ verwendet werden, wird im hochschulischen Kontext mit den Begriffen der Anerkennung und der Anrechnung operiert. Da sich der vorliegende Beitrag der Fragestellung am Beispiel des Feldes der wissenschaftlichen Weiterbildung annähert, wird an dieser Stelle eine genauere Bestimmung der Begrifflichkeiten vorgenommen. Dies ist insbesondere vor dem Hintergrund notwendig, da sich in den vergangenen Jahren zwei unterschiedliche Verständnisse zur Definition von Anerkennung und Anrechnung herausgebildet haben.

\footnotetext{
5 Für ausführliche Begriffsdefinitionen siehe Europäisches Zentrum für die Förderung der Berufsbildung (Cedefop) (2009).
} 
Tab. 1 Systematik der Definitionen von Anerkennung und Anrechnung. (Damm 2018, S. 21)

\begin{tabular}{|c|c|c|}
\hline- & Systemorientiert & Prozessorientiert \\
\hline Anerkennung & $\begin{array}{l}\text { Leistungen aus dem Hochschulsys- } \\
\text { tem (Akkreditierungsrat 2016) }\end{array}$ & $\begin{array}{l}\text { Prüfung (außer-)hochschulischer Vorleistun- } \\
\text { gen auf Gleichwertigkeit zu Studienleistun- } \\
\text { gen (Hanak und Sturm 2015b) }\end{array}$ \\
\hline Anrechnung & $\begin{array}{l}\text { Außerhochschulische Vorleistungen } \\
\text { (Akkreditierungsrat 2014) }\end{array}$ & $\begin{array}{l}\text { Vorgang des Ersetzens von Studien- durch } \\
\text { Vorleistungen (Hanak und Sturm 2015b) }\end{array}$ \\
\hline
\end{tabular}

Das systemorientierte Verständnis operiert bei innerhalb des Hochschulsystems erworbenen Kompetenzen mit dem Begriff Anerkennung und bei außerhalb des Hochschulsystems erworbenen Kompetenzen mit dem Begriff Anrechnung. Bei beiden Begrifflichkeiten ist nach diesem Verständnis sowohl der Prozess der Feststellung der Gleichwertigkeit, als auch der Ersetzung von Studieninhalten inkludiert. ${ }^{6}$

Das prozessorientierte Verständnis ordnet die beiden Begrifflichkeiten den übergeordneten Prozessschritten des Verfahrens zu. In einem ersten Schritt sind die bereits erworbenen Kompetenzen (unabhängig davon, ob sie im hochschulischen oder im außerhochschulischen Kontext erworben wurden) mit den Kompetenzen auf Gleichwertigkeit hin zu prüfen, die sie ersetzen sollen. Eine positive Überprüfung nach Inhalt und Niveau führt zu einer Anerkennung der Gleichwertigkeit. In einem zweiten Schritt können die zuvor als gleichwertig anerkannten Kompetenzen genutzt werden, um sie mit dem Ziel der Zulassung zu Studienprogrammen oder der Reduzierung des zu studierenden Workloads anzurechnen. ${ }^{7}$ Eine entsprechende Differenzierung der Referenzsysteme, in denen die Kompetenzen erworben wurden, wird nach dem prozessorientierten Verständnis durch die Begriffe hochschulisch bzw. außerhochschulisch erworbene Kompetenzen vorgenommen. Tab. 1 visualisiert unterstützend die differierenden Systematisierungen der Begriffe Anerkennung und Anrechnung.

Der vorliegende Beitrag greift aus unterschiedlichen Gründen auf das prozessorientierte Verständnis zurück. Zum einen werden diese Begriffsdefinitionen im überwiegenden Teil der wissenschaftlichen Auseinandersetzung mit der Thematik zugrunde gelegt (vgl. Birker und Damm 2015; Hanak und Sturm 2015a,b; Hanft et al. 2014; Luft et al. 2018; Maier 2018). Zum anderen ist dieses Begriffsverständnis besonders dienlich für eine differenzierte Auseinandersetzung mit der diesem Beitrag zugrunde liegenden Fragestellung der Passungsprobleme zwischen formaler und institutioneller Anerkennung in (erwachsenen-)pädagogischen Kontexten.

Daher wird „Anerkennung“ im Folgenden als Prüfung (außer-)hochschulisch erworbener Kompetenzen auf ihre Gleichwertigkeit zu den in einem Studienprogramm vermittelten Kompetenzen und „Anrechnung“ als Vorgang des Ersetzens einer oder

\footnotetext{
${ }^{6}$ Ein weiteres Ergebnis dieses Prozesses, neben der Ersetzung von Studieninhalten und der damit verbundenen Reduzierung des zu studierenden Workloads, kann auch die Zulassung zu Studienprogrammen oder (bei kostenpflichtigen Programmen) die Reduktion der Teilnehmendenentgelte sein. Für umfassende Darstellungen zu den Funktionen von Anrechnung siehe Hanak und Sturm (2015a, S. 23-25).

7 Auch eine so genannte ,doppelte Anrechnung“ ist möglich. Eine als gleichwertig anerkannte Kompetenz kann demnach sowohl für die Zulassung als auch zur Reduzierung des zu studierenden Workloads genutzt (angerechnet) werden.
} 
mehrerer Studien- und Prüfungsleistungen durch (außer-)hochschulisch erworbene Kompetenzen, die zuvor als gleichwertig anerkannt wurden, verstanden.

\section{Passungsprobleme zwischen formaler und institutioneller Anerkennung}

Im Folgenden wird unter Rückgriff auf das Feld der wissenschaftlichen Weiterbildung untersucht, inwiefern Passungsprobleme zwischen der formalen und der institutionellen Anerkennung zu Übersetzungsproblemen der durch bereits vorliegende Verfahren validierten Kompetenzen in den hochschulischen Kontext führen. Dabei lassen sich die in der Anrechnungsliteratur verfügbaren empirischen Ergebnisse in Bezug auf Passungsprobleme drei relevanten Ebenen zuordnen.

Auf der operativen Ebene werden administrative Aspekte beleuchtet (wie etwa ungeklärte Prozesse und Verantwortlichkeiten), die einer Anerkennung entgegenstehen können. Auf der strategischen Ebene werden mögliche Konsequenzen der Anerkennung non-formal und informell erworbener Kompetenzen für die Bildungsorganisation (wie etwa eine strategische Öffnung oder Schließung gegenüber neuen Zielgruppen) betrachtet. Und auf der organisationskulturellen Ebene werden habituelle Aspekte (wie etwa Modi der Abwehr) gegen die institutionelle Anerkennung außerhalb der Bildungseinrichtung erworbener Kompetenzen fokussiert (Abschn. 4).

\subsection{Operative Ebene}

Um der Annahme nachzugehen, dass organisationale Implementierungshemmnisse von Verfahren zur Anerkennung und Anrechnung außerhochschulisch erworbener Kompetenzen innerhalb der jeweiligen hochschulischen Prozesse verortet sind, haben Sturm und Hanak (2018) eine generelle Systematisierung von Anrechnungsprozessen entwickelt. Abb. 1 zeigt vier Kernprozesse von Anrechnungsverfahren.

Diese Bestimmung von Kernprozessen bietet eine Grundlage für vertiefende Betrachtungen auf der Prozessebene und eröffnet dadurch die Möglichkeit, Passungsprobleme zu identifizieren, die ihre Wirkung auf der operativen Ebene des einzelnen Prozessschrittes entfalten. Um mögliche Passungsprobleme zwischen der formalen und der institutionellen Anerkennung außerhochschulisch erworbener Kompetenzen zu identifizieren, wird im Folgenden exemplarisch ein Prozessschritt herausgegriffen. Ausgehend von der Systematisierung in vier Kernprozesse und geleitet von einer problemzentrierten Vorgehensweise hat Sturm (2018a) den ersten Prozessschritt mit dem Ziel untersucht, Optimierungspotentiale auf dem Feld der Anrechnungsberatung

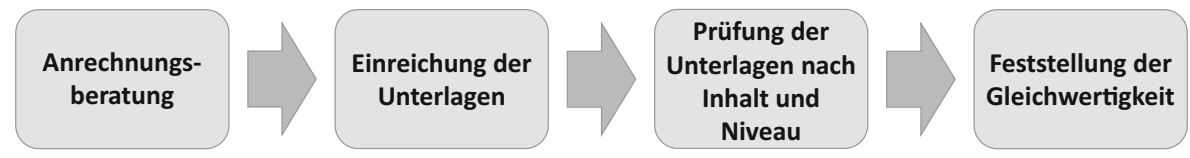

Abb. 1 Kernprozesse von Anrechnungsverfahren. (Sturm und Hanak 2018, S. 186) 
zu identifizieren. ${ }^{8}$ Diese Betrachtungen haben gezeigt, dass für die Anrechnungsberatung ,bisher kaum formalisierte intraorganisationale Strukturen ausgebildet wurden“ (Sturm 2018a, S. 293). Beim Aufbau dieser Strukturen ist insbesondere darauf zu achten, den bisher schwach ausgeprägten und wenig transparenten Organisations- und Ablaufstrukturen entgegenzuwirken und die für die Anrechnungsberatung relevanten Wissensträgerinnen und -träger gezielt miteinander zu vernetzen, um dadurch die notwendigen Kommunikations- und Kooperationsprozesse zu initiieren (vgl. Sturm 2018a, S. 294). Darüber hinaus sind Informationen zum Ablauf von Anrechnungsverfahren für alle am Prozess beteiligten Akteurinnen und Akteure zentral aufzubereiten, strukturiert weiterzugeben und dauerhaft abrufbar zu machen (vgl. ebd., S. 294). Zudem könnte eine klar definierte Kontaktperson oder Organisationseinheit sowohl die Kooperations- und Kommunikationsstrukturen organisieren als auch ein zentrales Wissensmanagement zur Anrechnung außerhochschulisch erworbener Kompetenzen betreuen (vgl. ebd.).

Voraussetzung dafür, dass diese Maßnahmen zu einer Reduzierung von Passungsproblemen zwischen der Feststellung der Gleichwertigkeit außerhochschulisch erworbener Kompetenzen und dem Einsatz dieser im hochschulischen Kontext beitragen, ist allerdings, dass das entsprechende Personal ausreichend geschult ist, um über das Anerkennungs- und Anrechnungsverfahren informieren zu können und über zeitliche Ressourcen verfügt, um dieser Aufgabe gerecht zu werden (vgl. Hanak und Sturm 2015a, S. 14).

Die exemplarische Betrachtung eines ausgewählten Prozessschrittes im Rahmen von Anerkennungs- und Anrechnungsverfahren zeigt, dass schwach ausgeprägte intraorganisationale Strukturen auf operativer Ebene zu Übersetzungsproblemen der validierten Kompetenzen in den hochschulischen Kontext führen. Eine intensive Untersuchung der weiteren Prozessschritte, sowie die Operationalisierung der sich daraus ergebenden Erkenntnisse in eine organisationale Handlungspraxis erscheinen als notwendige Voraussetzungen, sollen aufwendig entwickelte und durchgeführte Verfahren zur Anerkennung und Anrechnung außerhochschulisch erworbener Kompetenzen ihre volle Wirkung entfalten und zu einer Intensivierung der Anrechnungspraxis an Hochschulen beitragen.

\subsection{Strategische Ebene}

Auch strategische organisationsbezogene Überlegungen wirken auf den institutionellen Umgang mit Anerkennung und Anrechnung außerhochschulisch erworbener Kompetenzen. Damm (2018) identifiziert anhand qualitativ-empirischer Analysen vier unterschiedliche hochschulische Handlungsmuster.

Im Handlungsmuster erforderliche Begrenzung spielt Anrechnung eine untergeordnete Rolle. Das wesentliche Ziel besteht darin, die weiterbildenden Studienprogramme in ihrer aktuellen Form zu erhalten. Zu beobachten ist diese Strategie bei Studienprogrammen, in denen die Bewerbendenzahlen konstant oder höher als erfor-

\footnotetext{
8 Hierzu wurden 18 Experteninterviews mit Beratenden auf unterschiedlichen Ebenen (Hochschul-, Fachbereichs- und Studienprogrammebene) an drei Hochschulen geführt. Für weiterführende Informationen siehe Sturm (2018a).
} 
derlich sind (vgl. Damm 2018, S. 62). Um den Status quo möglichst zu konservieren, werden in Folge hochschulrechtlicher Forderungen zwar formal Anrechnungsmöglichkeiten geschaffen. Diese werden in der praktischen Umsetzung allerdings auf ein notwendiges $\mathrm{Ma} ß$ beschränkt und so organsiert, dass begründet werden kann, warum eine Anrechnung über die bisher gebotenen Bereiche hinaus nicht möglich ist (vgl. ebd., S. 59). Anrechnung kann in diesem Handlungsmuster als ein notwendiges Mittel zum Zweck betrachtet werden, um bestimmte Passungsprobleme, wie das Schließen der Bachelor-Master-Lücke, ${ }^{9}$ handhabbar zu machen, ohne dass die bisherigen Grenzen der Angebote verschoben werden (vgl. ebd., S. 61).

Das Handlungsmuster der selbstverständlichen Exklusivität kann in weiterbildenden Studienprogrammen beobachtet werden, die primär dem Erwerb von Titeln dienen und weniger der Erweiterung von Kenntnissen. Der Wert der Abschlüsse hängt dabei wesentlich davon ab, wie exklusiv der Zugang zu dem Studienprogramm ist. Daher ist es für diese Programme wichtig, die Zulassungshürden entsprechend hoch anzulegen (vgl. ebd., S. 64). Im Gegensatz zum Handlungsmuster der erforderlichen Begrenzung wird nicht versucht die Bachelor-Master-Lücke auszugleichen, vielmehr dient diese als gewolltes Zulassungshindernis (vgl. ebd., S. 66). Die Anrechnung von außerhochschulischen Vorleistungen mit dem Ziel der Verkürzung des zu studierenden Workloads hat in diesem Handlungsmuster die Funktion, die Attraktivität der Studienprogramme zu erhöhen (vgl. ebd., S. 67). Dabei werden nur Vorleistungen anerkannt, die aufwändig zu erwerben sind, wie beispielsweise umfangreiche und spezialisierte Berufserfahrung (vgl. ebd., S. 67).

Charakteristisch für Studienprogramme, die das Handlungsmuster der gezielt genutzten Öffnung repräsentieren, sind junge interdisziplinäre Angebote oder Akademisierungsmöglichkeiten im Anschluss an Berufsausbildungen (vgl. ebd., S. 68). Dabei wird die Möglichkeit der Zulassung für nicht-traditionelle Zielgruppen ohne klassische Hochschulzugangsberechtigung gezielt genutzt, um Teilnehmende für bisher wenig etablierte Angebote zu rekrutieren. Neben der dadurch erreichten Auslastung des Angebots kann die Finanzierung der, in der Regel befristeten, Mitarbeitendenstellen ${ }^{10}$ gesichert werden. Zentrale Zielgruppe für die Anerkennung und Anrechnung mit dem Ziel der Zulassung zu diesen Studienprogrammen sind Bewerbende, ,die in einer der Bezugsdisziplinen eines interdisziplinären Fachs oder einem zu akademisierenden Berufsfeld umfassende und einschlägige berufliche Erfahrungen gesammelt haben“" (ebd., S. 73). Eine Anrechnung mit dem Ziel der Ersetzung von Studieninhalten ist in diesem Handlungsmuster in der Regel nicht vorgesehen, da berufspraktische Erfahrungen didaktisch in die Studienprogramme eingebettet werden, wodurch ein zentrales Qualitätsmerkmal erzeugt wird (vgl. ebd., S. 74).

\footnotetext{
9 Die Bachelor-Master-Lücke beschreibt die Anzahl der fehlenden ECTS-Punkte, die nach einem abgeschlossenem Bachelor-Studium notwendig ist, um ein (weiterbildendes) Masterstudium aufnehmen zu können, welches mit 300 ECTS-Punkten abschließt.

${ }^{10}$ Hierbei handelt es sich um Hochschulprofessionelle, welche die weiterbildenden Studienangebote betreuen. Die genaue Berufsbezeichnung variiert zwischen verschiedenen Programmen und Hochschulen. Oft genutzte Bezeichnungen sind Studiengangskoordinierende, Studiengangsmanagerinnen und -manager oder Geschäftsführerinnen und -führer. Für weiterführende Informationen siehe Rundnagel (2018).
} 
Im Handlungsmuster der zentral gewollten Öffnung sind die Themen der Anerkennung und Anrechnung außerhochschulisch erworbener Kompetenzen zentral auf der Ebene der Hochschulleitungen angesiedelt. Zudem verfügen Hochschulen, die diesem Handlungsmuster zuzuordnen sind, meist über zentrale Organisationseinheiten zur Steuerung der wissenschaftlichen Weiterbildung (vgl. ebd., S. 74). Im Kontrast $\mathrm{zu}$ den anderen drei Handlungsmustern ist nicht die jeweilige Profession die Bezugsgröße für den Umgang mit der Anerkennung und Anrechnung außerhochschulisch erworbener Kompetenzen, sondern die gesamte Organisation mit ihren Bemühungen der Etablierung der wissenschaftlichen Weiterbildung als dritte Säule neben Forschung und Lehre. Dabei wird davon ausgegangen, dass jedes weiterbildende Studienprogramm für neue Zielgruppen geöffnet werden kann, ohne das eigene Profil zu verlieren (vgl. ebd., S. 80). Diese strategische Öffnung hin zu einem Weiterbildungsmarkt stellt Hochschulen vor die Herausforderung, sich zwischen der Nutzung der Instrumente der Anrechnung mit dem Ziel der Gewinnung neuer Zielgruppen auf der einen Seite und den damit verbundenen Risiken der Kostenreduktion auf der anderen Seite bewegen zu müssen. Führt die Anrechnung von Kompetenzen dazu, dass die Durchführungskosten nicht mehr gedeckt werden können, ,muss möglichst inhaltlich begründet werden, warum es für die einzelnen [Teilnehmenden] besser sei, das gesamte Programm zu studieren - was voraussetzt, dass angerechnete Module nicht bezahlt werden müssen“ (ebd., S. 78).

Die Gesamtschau der vier Handlungsmuster zeigt, dass der Einsatz von Instrumenten der Anerkennung und Anrechnung keineswegs ausschließlich von rechtlichen Rahmenbedingungen oder verfügbaren Verfahren bestimmt wird. Vielmehr spielen komplexe professions- oder hochschulstrategische Überlegungen eine wesentliche Rolle dabei, in welchem Umfang und mit welchem Ziel von den Möglichkeiten der Anrechnung Gebrauch gemacht wird.

\subsection{Organisationskulturelle Ebene}

Im Rahmen einer Praxisanalyse haben Hanak und Sturm (2015b) die mangelnde Akzeptanz gegenüber der Gleichwertigkeit von außerhochschulisch erworbenen zu hochschulisch vermittelten Kompetenzen als einen wesentlichen Grund ${ }^{11}$ für eine geringe Anrechnungspraxis herausgestellt. Dabei wird von Seiten der Hochschulen insbesondere die Sorge artikuliert, ,in den Ruf zu geraten, es würde den Studierenden alles geschenkt" (Hanak und Sturm 2015b, S. 78). Daran anknüpfend hat Sturm (2016) anhand vertiefender Betrachtungen herausgearbeitet, dass ein wesentlicher Faktor für die geringe Übersetzungsquote validierter Kompetenzen in den akademischen Kontext auf organisationskultureller Ebene in der ausstehenden Debatte über den Stellenwert von akademischer Bildung und ihrer Abgrenzung zu außerhochschulischer Bildung gesehen werden muss (vgl. Sturm 2016, S. 64). Die Unklarheit darüber, wodurch sich der Wert akademischer Bildung kennzeichnet, führt zu organisationskulturellen Widerständen und somit zu Modi habitueller Abwehr

\footnotetext{
11 Die Autoren haben insgesamt sechs zentrale Aspekte identifiziert, deren Bearbeitung zu einer Erhöhung der Akzeptanz gegenüber der Anrechnungsthematik innerhalb von Hochschulen führt. Für umfassende Darstellungen siehe Hanak und Sturm (2015b).
} 
gegenüber der Anerkennung der Gleichwertigkeit außerhochschulisch erworbener Kompetenzen. Erst wenn Klarheit darüber hergestellt wird, wo die Schnittmengen und, damit verbunden, auch die Abgrenzungen zwischen akademischer und außerhochschulischer (beispielsweise beruflicher) Bildung verlaufen, kann eine Sicherung des akademischen Niveaus im Rahmen von Anerkennungs- und Anrechnungsverfahren vorgenommen werden. Solange diese Klarheit nicht hergestellt ist, stellt das außerhochschulisch erworbene Wissen im akademischen Raum ein wesentliches Hemmnis bei der Implementierung von Anerkennungs- und Anrechnungsverfahren dar (vgl. ebd., S. 65).

Zusammenfassend betrachtet bedarf es umfassender organisationskultureller Vorarbeiten und Klärungsprozesse auf verschiedenen Ebenen und unter Berücksichtigung der organisationalen Spezifika von Hochschulen, ${ }^{12}$ um die Voraussetzungen dafür zu schaffen, dass entwickelte und erprobte Anrechnungsverfahren im Rahmen der rechtlichen Leitplanken in den Hochschulen implementiert und nachhaltig verankert werden können (vgl. Sturm 2018b, S. 133).

\section{Ergebnisdarstellung}

Im Rahmen der Ergebnisdarstellung wird in einem ersten Schritt eine kombinatorische Gesamtschau der drei untersuchten Ebenen vorgenommen, um daraus organisationsbedingte Passungsprobleme ableiten zu können. In einem zweiten Schritt werden die Ergebnisse auf die Begriffsarchitektur des Feldes reflektiert um hier entsprechende Leerstellen zu beleuchten und ergänzende Vorschläge zu unterbreiten.

\subsection{Zusammenschau der Passungsprobleme}

In der Gesamtbetrachtung der drei aus der Anrechnungsliteratur abgeleiteten Ebenen, mit dem Fokus auf Passungsprobleme zwischen formaler und institutioneller Anerkennung als Erklärungsversuch für die geringe organisationale Verankerung in der deutschen Hochschullandschaft, wird deutlich, dass es nicht primär weiterer Ausdifferenzierungen von Verfahren zur Anerkennung und Anrechnung außerhochschulisch erworbener Kompetenzen bedarf, um die Thematik breit und nachhaltig in den Hochschulstrukturen zu verankern. Vielmehr bedarf es organisationaler Vorarbeiten auf den drei dargestellten Ebenen.

So gilt es auf der operativen Ebene mit Blick auf die dargestellten Kernprozesse von Anrechnungsverfahren (Abb. 1) entsprechende Strukturen und Verfahrenspraktiken zu schaffen und zu institutionalisieren. Auf der strategischen Ebene ist zu klären, welche strategische Funktion die Anerkennungspraxis für die jeweilige Hochschule auf Organisations-, Fachbereichs- oder Programmebene einnehmen soll (zentral gewollte Öffnung, gezielt genutzte Öffnung, erforderliche Begrenzung oder selbstverständliche Exklusivität). Auf der organisationskulturellen Ebene bedarf es

12 Organisationale Spezifika von Hochschulen finden sich beispielsweise auf der Ebene der Hochschulleitungen und der Professorinnen und Professoren. Für weitere Betrachtungen siehe Sturm (2018b, S.128-131). 
kultureller Vorarbeiten in Bezug auf die Selbstvergewisserung des Wertes akademischer Bildung in Abgrenzung zu außerhalb des Hochschulkontextes erworbener Kompetenzen sowie einer Auseinandersetzung mit der Frage, welche Bedeutung außerhochschulisch erworbenem Wissen im akademischen Raum zugeschrieben wird. Dabei sollten die drei Ebenen nicht isoliert voneinander betrachtet werden. Vielmehr muss hierbei von gegenseitigen Abhängigkeiten und Einflussnahmen ausgegangen werden.

Demnach wird eine Hochschule, die eine Strategie der gezielt genutzten Öffnung verfolgt, auf der operativen Ebene stärkere Anstrengungen unternehmen, entsprechende Kommunikations- und Kooperationsstrukturen $\mathrm{zu}$ entwickeln als eine Hochschule, die strategisch eine erforderliche Begrenzung betreibt. Auch wird die Bereitschaft organisationskulturelle Klärungsprozesse $\mathrm{zu}$ initiieren bei Hochschulen stärker ausgeprägt sein, in denen die Hochschulleitung anhand einer zentral gewollten Öffnung die Anrechnungsthematik, im Idealfall katalysiert durch entsprechende Drittmittelprojekte, offensiv nutzt, um Weiterbildung als dritte Säule neben Forschung und Lehre in die Hochschulstrukturen zu implementieren. Um Passungsprobleme zwischen der formalen Anerkennung (anhand vorliegender Verfahren) und der institutionellen Anerkennung (durch die Hochschulen) zu identifizieren und entsprechende Maßnahmen mit dem Ziel einzuleiten, eine Übersetzung der bereits als gleichwertig anerkannten außerhochschulisch erworbenen Kompetenzen in den akademischen Raum zu organisieren, bedarf es einer gleichzeitigen Betrachtung und Berücksichtigung aller drei Ebenen.

Abb. 2 bettet die Erkenntnisse aus der Analyse von Passungsproblemen zwischen formaler und institutioneller Anerkennung in die Logik eines übergeordneten Prozesses ein. Demnach erfolgt, unter Rückgriff auf bereits vorliegende Verfahren und Instrumente, in einem ersten Schritt die Anerkennung der Gleichwertigkeit der außerhochschulisch erworbenen Kompetenzen. In einem zweiten Schritt gilt es nun die als gleichwertig anerkannten Kompetenzen in den institutionellen Kontext von Hochschulen zu übersetzen. In einem dritten Schritt können die als gleichwertig anerkannten und organisational übersetzten Kompetenzen, wiederum anhand vorliegender Instrumente und Verfahren, angerechnet werden, um so den Zugang zu

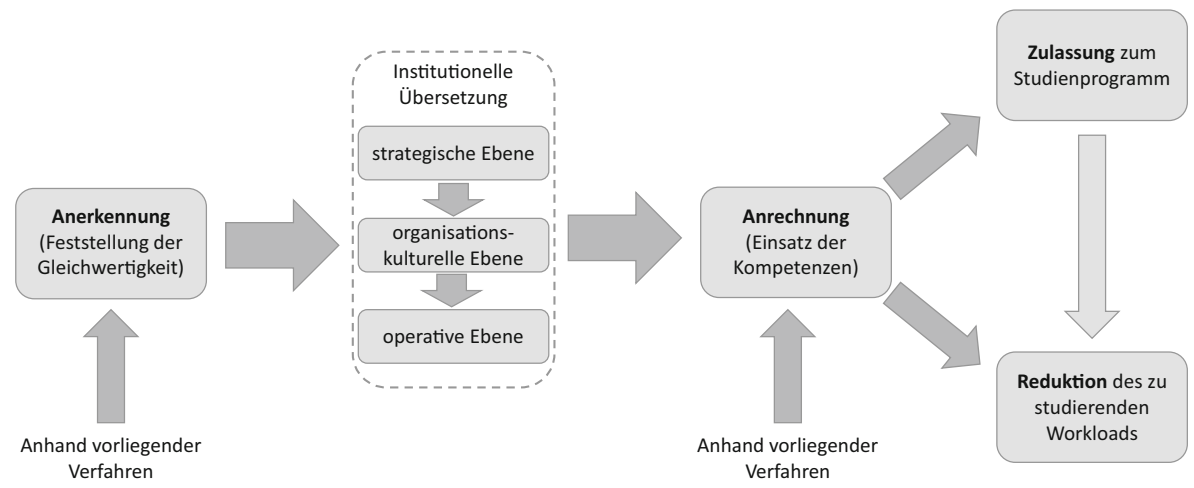

Abb. 2 Erweiterung der Darstellung von Anerkennungs- und Anrechnungsverfahren. (Eigene Darstellung) 
Studienprogrammen zu ermöglichen oder den zu studierenden Workload zu reduzieren.

Die zentralen Erkenntnisse aus der gleichzeitigen Gesamtschau der drei untersuchten Ebenen bestehen zum einen darin, dass Passungsprobleme zwischen formaler und institutioneller Anerkennung bedeutsame Hemmnisse für die nachhaltige organisationale Verankerung von Anrechnungsverfahren darstellen. Zum anderen wird sichtbar, dass der Prozess der Übersetzung der formal anerkannten Kompetenzen in den institutionellen Kontext der Zielorganisation bisher kaum Beachtung gefunden hat, obwohl ihm eine zentrale Bedeutung für die nachhaltige organisationale Verankerung von Anrechnungsverfahren zukommt.

\subsection{Leerstellen in der Begriffsarchitektur}

Die Erkenntnis, dass der Prozess der organisationalen Übersetzung kaum tiefergehende Aufmerksamkeit erfahren hat, dokumentiert sich auch in Form einer Leerstelle in der Begriffsarchitektur des Feldes. Während den Prozessen der Feststellung der Gleichwertigkeit (Anerkennung) und der Nutzung der außerhochschulisch erworbenen Kompetenzen zur Zulassung zu Studienprogrammen oder zur Reduzierung des zu studierenden Workloads (Anrechnung) klare Begrifflichkeiten zugeordnet sind, ${ }^{13}$ ist der Prozess der Übersetzung von Kompetenzen in den institutionellen Kontext der Zielorganisation begrifflich bisher nicht gefasst und in keinen Verfahrensablauf eingebettet. Um die gleichwertige Bedeutung des Prozessschrittes der institutionellen Übersetzungsleistung - mit den Schritten der Anerkennung und Anrechnung kenntlich zu machen, wird daher eine Erweiterung der bisherigen Begriffsarchitektur vorgeschlagen. Wie in Abb. 3 dargestellt, folgt auf die Anerkennung zunächst die Translation, bevor innerhalb der Zielorganisation eine Anrechnung erfolgen kann.

Durch diese Erweiterung der Begriffsarchitektur gewinnt das Feld der organisationalen Übersetzung, und damit der Reduzierung von Passungsproblemen zwischen formaler und institutioneller Anerkennung, wesentlich an Bedeutung. Erfährt dieser Aspekt ebenso viel Aufmerksamkeit wie der Entwicklung von Verfahren zur Anerkennung und Anrechnung in der vergangenen Dekade zuteil wurde, würde dies einen wesentlichen Beitrag zur organisationalen Befähigung zur Implementierung von Anrechnungsverfahren bedeuten. Ziel dabei wäre explizit nicht die konsequente Öffnung von Hochschulen für nicht-traditionell Studierende oder die Schaffung von möglichst umfangreichen Potentialen zur Reduzierung des zu studierenden Workloads. Vielmehr könnten Hochschulen Anrechnung entsprechend ihrer individuellen operativen, strategischen und organisationskulturellen Selbstverständnisse proaktiv gestalten.

\footnotetext{
${ }^{13}$ Hierbei wird das prozessorientierte Verständnis der Begriffe Anerkennung und Anrechnung zugrunde gelegt. Siehe hierfür Abschn. 2.
} 


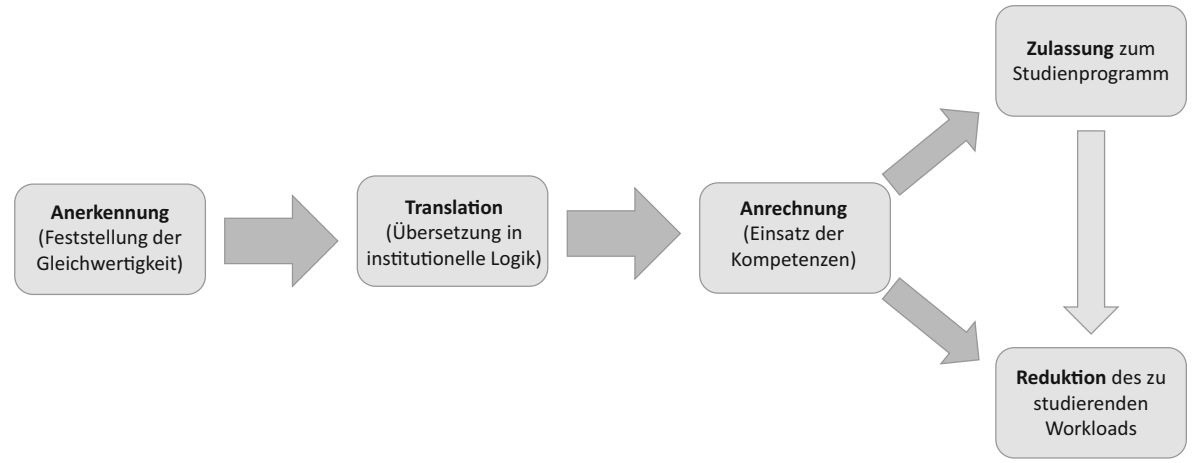

Abb. 3 Neuformatierung der Begriffsarchitektur von Anerkennungs- und Anrechnungsverfahren. (Eigene Darstellung)

\section{Rückbindung auf andere (erwachsenen-)pädagogische Kontexte}

Am Beispiel des Feldes der wissenschaftlichen Weiterbildung wurden Passungsprobleme zwischen der formalen Anerkennung (durch die vorliegenden Verfahren) und der institutionellen Anerkennung (durch die jeweilige Hochschule) auf drei Ebenen identifiziert. Gleichzeitig konnte eine Leerstelle in der Begriffsarchitektur des Feldes benannt werden, die eine zielgerichtete Bearbeitung von Translationsproblemen in den Kontext der anrechnenden Zielorganisation erschwert.

Aus diesen Betrachtungen lässt sich ableiten, dass der geringe Verbreitungsgrad der Anerkennung und Anrechnung außerhochschulisch erworbener Kompetenzen nicht durch einen Mangel an praktikablen, validen und reliablen Verfahren zur Validierung non-formaler und informeller Kompetenzen zu erklären ist. Vielmehr scheint die schwache organisatorische Translationsleistung von bereits validierten Kompetenzen in dem jeweiligen institutionellen Kontext der anerkennenden Zielorganisation das zentrale Hemmnis für eine flächendeckende Implementierung entsprechender Verfahren darzustellen. Diese Erkenntnis bietet interessante Potentiale für die Generalisierung und Adaptierung auf andere (erwachsenen-)pädagogische Kontexte. Exemplarisch soll dies am Beispiel der beruflichen Bildung skizziert werden. Im Unterschied zur hochschulischen Bildung mit ihrer Begriffsarchitektur (Anerkennung, Anrechnung) bietet die berufliche Bildung eine begriffliche Bestimmung für drei Phasen (Ermittlung, Validierung und Zertifizierung) ${ }^{14}$ des Verfahrens an. Gemäß der Definition des europäischen Zentrums für die Förderung der Berufsbildung bleibt jedoch auch hier der Prozess der Translation (Übersetzung in die institutionelle Logik) eine begriffliche Leerstelle. ${ }^{15}$ Abb. 4 setzt die Begriffsarchitektur der beruflichen und der hochschulischen Bildung in ein Verhältnis zueinander und zeigt,

\footnotetext{
${ }^{14}$ Im Zusammenhang von Validierung wird teilweise auch der Begriff Anerkennung verwendet (vgl. Europäisches Zentrum für die Förderung der Berufsbildung (Cedefop) 2009, S. 85).

15 Die ausführlichen Begriffsdefinitionen sind in den europäischen Leitlinien für die Validierung nicht formalen und informellen Lernens dokumentiert (vgl. Europäisches Zentrum für die Förderung der Berufsbildung (Cedefop) 2009).
} 


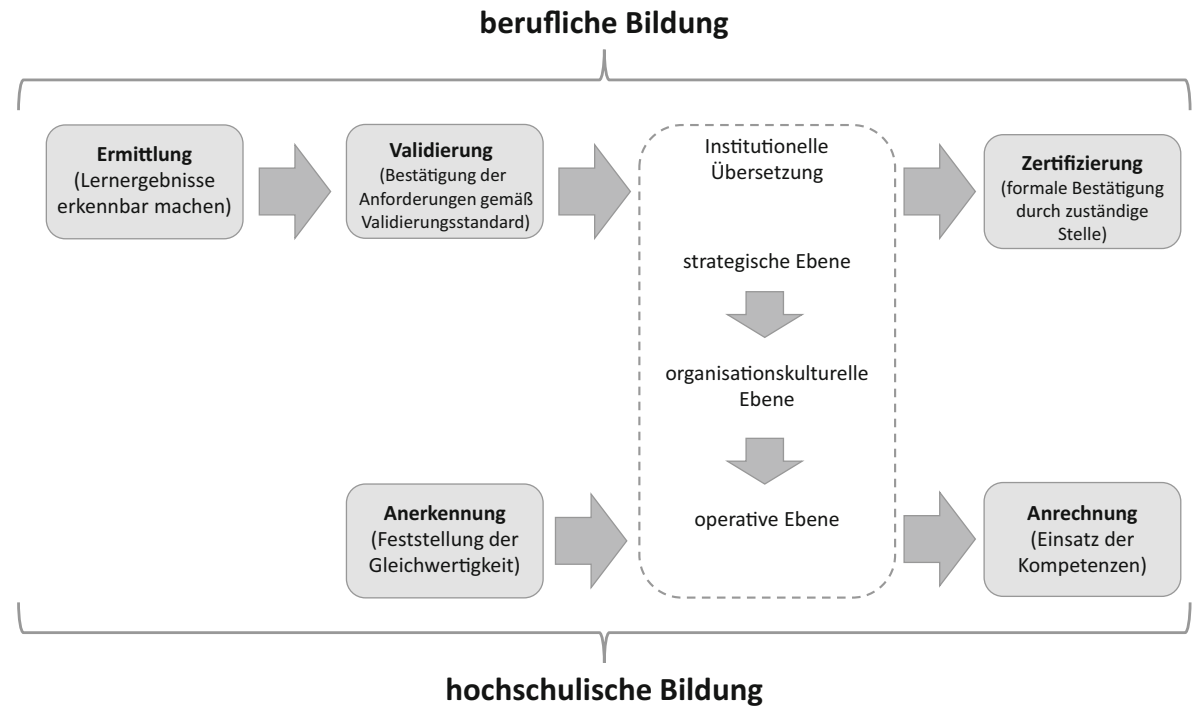

Abb. 4 Gegenüberstellung der Begriffsarchitekturen. (Eigene Darstellung)

dass sich die auf dem Feld der hochschulischen Bildung identifizierte Leerstelle in der Begriffsarchitektur auch auf dem Feld der beruflichen Bildung wiederfindet. Im Unterschied zur hochschulischen Bildung bietet die berufliche Bildung mit der Explikation der „Ermittlung“ zwar einen weiteren Prozessschritt, in dem die Lernergebnisse einer Einzelperson festgehalten und erkennbar gemacht werden, der Prozess der Übersetzung in die eigene institutionelle Logik bleibt jedoch auch hier ausgeklammert.

Diese Erkenntnis kann zum Anlass genommen werden, um zu überprüfen, ob auch auf dem Feld der beruflichen Bildung eine Schwäche in der organisatorischen Translationsleistung von bereits validierten Kompetenzen in den eigenen institutionellen Kontext als hemmender Faktor für eine Anrechnung hochschulisch erworbener Kompetenzen identifiziert werden kann. Davon ausgehend könnten, durch Rückbindung auf die am Beispiel der wissenschaftlichen Weiterbildung identifizierten Ebenen, mögliche Passungsprobleme sichtbar gemacht werden. Insbesondere vor dem Hintergrund, dass im Prozess der institutionellen Übersetzung verfahrenshinderliche Faktoren vermutet werden müssen, ist vertiefenden Betrachtungen auf organisationskultureller Ebene eine besondere Bedeutung beizumessen. Auf diese Weise wäre zu ermitteln, ob Passungsprobleme zwischen formaler und institutioneller Anerkennung in beiden Referenzsystemen einer wechselseitigen Durchlässigkeit entgegenstehen.

Aber auch in Bezug auf weitere (erwachsenen-)pädagogische Zusammenhänge kann überprüft werden, ob Passungsprobleme zwischen formaler und institutioneller Anerkennung vorliegen und welche Rahmenbedingungen bzw. Vorarbeiten auf den identifizierten Ebenen notwendig sind, um die organisationale Translationsfähigkeit zu erhöhen. 
Open Access Dieser Artikel wird unter der Creative Commons Namensnennung 4.0 International Lizenz (http://creativecommons.org/licenses/by/4.0/deed.de) veröffentlicht, welche die Nutzung, Vervielfältigung, Bearbeitung, Verbreitung und Wiedergabe in jeglichem Medium und Format erlaubt, sofern Sie den/die ursprünglichen Autor(en) und die Quelle ordnungsgemäß nennen, einen Link zur Creative Commons Lizenz beifügen und angeben, ob Änderungen vorgenommen wurden.

\section{Literatur}

Akkreditierungsrat (2014). Anrechnung außerhochschulischer Kenntnisse und Fähigkeiten. http://www. akkreditierungsrat.de/fileadmin/Seiteninhalt/KMK/Vorgaben/KMK_Anrechnung_ausserhochschu lisch_II.pdf. Zugegriffen: 15.10.2018.

Akkreditierungsrat (2016). Anwendung der Lissabon-Konvention. http://archiv.akkreditierungsrat.de/ fileadmin/Seiteninhalte/AR/Beschluesse/AR_Lissabon_Konvention.pdf. Zugegriffen: 15.10.2018.

Birker, A., \& Damm, C. (2015). Anrechnung außerhochschulischer Vorleistungen. Hochschulinterne Broschüre für Studiengangsentwickler_innen der Hochschule Magdeburg-Stendal und der Ottovon-Guericke-Universität Magdeburg. http://www.weiterbildungscampus.de/weiterbildungscampus_ media/Dokumente/Anrechungsbrosch\%C3\%BCre+Weiterbildungscampus.pdf. Zugegriffen: 17. Mai 2018.

Damm, C. (2018). Anrechnung von außerhochschulischen Vorleistungen in der wissenschaftlichen Weiterbildung. Ergebnisse einer zweiteiligen empirischen Studie. Beiträge zur Weiterbildungsforschung, Bd. 1. Magdeburg: Otto-von-Guericke-Universität, Fakultät für Humanwissenschaften, Institut I: Bildung, Beruf und Medien.

Erpenbeck, J., Rosenstiel, L., Grote, S., \& Sauter, W. (2017). Handbuch Kompetenzmessung: Erkennen, verstehen und bewerten von Kompetenzen in der betrieblichen, pädagogischen und psychologischen Praxis (3. Aufl.). Stuttgart: Schäfer-Poeschel.

Europäische Kommission (2000). Memorandum über Lebenslanges Lernen. Brüssel: Kommission der Europäischen Gemeinschaften.

Europäisches Zentrum für die Förderung der Berufsbildung (2009). Europäische Leitlinien für die Validierung nicht formalen und informellen Lernens. Luxemburg: Amt für Veröffentlichungen der Europäischen Union.

Europäisches Zentrum für die Förderung der Berufsbildung (2016). Arbeitsprogramm 2016. Luxemburg: Amt für Veröffentlichungen der Europäischen Union.

Faulstich, P., Graessner, G., \& Bade-Becker, U. (2007). Länderstudie Deutschland. In A. Hanft \& M. Knust (Hrsg.), Internationale Vergleichsstudie zur Struktur und Organisation der Weiterbildung an Hochschulen (S. 84-188). Oldenburg: Universität Oldenburg.

Federal Institute for Vocational Education and Training (2016). Germany. VET in Europe-2016 country report. Bielefeld: W. Bertelsmann.

Freitag, W., Buhr, R., Danzeglocke, E., Schröder, S., \& Völk, D. (Hrsg.). (2015). Übergänge gestalten. Durchlässigkeit zwischen beruflicher und hochschulischer Bildung erhöhen. Münster: Waxmann.

Hanak, H., \& Sturm, N. (2015a). Anerkennung und Anrechnung außerhochschulisch erworbener Kompetenzen. Eine Handreichung für die wissenschaftliche Weiterbildung. Wiesbaden: Springer VS.

Hanak, H., \& Sturm, N. (2015b). Außerhochschulisch erworbene Kompetenzen anrechnen. Praxisanalyse und Implementierungsempfehlungen. Wiesbaden: Springer VS.

Hanft, A., Brinkmann, K., Gierke, W. B., \& Müskens, W. (2014). Anrechnung außerhochschulischer Kompetenzen in Studiengängen. Studie: AnHoSt „Anrechnungspraxis in Hochschulstudiengängen“, Oldenburg. https://www.uni-oldenburg.de/fileadmin/user_upload/anrechnungsprojekte/Anhost.pdf. Zugegriffen: 17. Mai 2018.

Kultusministerkonferenz (2002). Anrechnung von außerhalb des Hochschulwesens erworbenen Kenntnissen und Fähigkeiten auf ein Hochschulstudium (I). Beschluss der Kultusministerkonferenz vom 28.06.2002. http://www.kmk.org/fileadmin/pdf/PresseUndAktuelles/2003/anrechnung.pdf. Zugegriffen: 31. Aug. 2017.

Kultusministerkonferenz (2008). Anrechnung von außerhalb des Hochschulwesens erworbenen Kenntnissen und Fähigkeiten auf ein Hochschulstudium (II). Beschluss der Kultusministerkonferenz vom 18.09.2008. http://www.kmk.org/fileadmin/veroeffentlichungen_beschluesse/2008/2008_09_ 18-Anrechnung-Faehigkeiten-Studium-2.pdf. Zugegriffen: 31. Aug. 2017.

Kultusministerkonferenz (2010). Ländergemeinsame Strukturvorgaben für die Akkreditierung von Bachelor- und Masterstudiengängen. Beschluss der Kultusministerkonferenz vom 10.10.2003 i.d.F. vom 
04.02.2010. http://www.kmk.org/fileadmin/Dateien/veroeffentlichungen_beschluesse/2003/2003_ 10_10-Laendergemeinsame-Strukturvorgaben.pdf. Zugegriffen: 31. Aug. 2017.

Kultusministerkonferenz (2011). Ländergemeinsame Strukturvorgaben für die Akkreditierung von Bachelor- und Masterstudiengängen vom 04.02.2010. Auslegungshinweise. http://www.akkreditierungsrat. de/fileadmin/Seiteninhalte/KMK/Vorgaben/KMK_Auslegungshinweise_Laendergemeinsame_ Strukturvorgaben.pdf. Zugegriffen: 31. Aug. 2017.

Londoner Kommuniqué (2007). Auf dem Wege zum Europäischen Hochschulraum: Antworten auf die Herausforderungen der Globalisierung. https://www.bmbf.de/files/Londoner_Kommunique_ Bologna_d.pdf. Zugegriffen: 22. Aug. 2018.

Luft, L., Schuler, N., Braun-Buss, A., Ambach, H., Schmidt, M., \& Kohlesch, A. (2018). Öffnung der Hochschule durch die Implementierung von Anrechnungsverfahren. In N. Sturm \& K. Spenner (Hrsg.), Nachhaltigkeit in der wissenschaftlichen Weiterbildung. Beiträge zur Verankerung in die Hochschulstrukturen (S. 141-164). Wiesbaden: Springer VS.

Maier, M.O. (2018). Intraorganisatorische Entwicklung der Beratung und Unterstützung an Hochschulen unter Bedingungen von Durchlässigkeit. In N. Sturm \& K. Spenner (Hrsg.), Nachhaltigkeit in der wissenschaftlichen Weiterbildung. Beiträge zur Verankerung in die Hochschulstrukturen (S. 165-186). Wiesbaden: Springer VS.

Müskens, W., Wittig, W., Tutschner, R., \& Eilers-Schoof, A. (2013). Module Level Indicator. MLI user guide-assessment of the level of competence orientation. Bremen: ITB.

Rundnagel, H. (2018). Studiengangkoordination in der wissenschaftlichen Weiterbildung als Gruppe der „,neuen“ Hochschulprofessionellen - zwischen Verwaltung und Wissenschaft? In T.C. Feld \& S. Lauber-Pohle (Hrsg.), Organisation und Profession. Felder erwachsenenpädagogischer Forschung (S. 217-234). Wiesbaden: Springer VS.

Schäfer, M., Kriegel, M., \& Hagemann, T. (Hrsg.). (2015). Neue Wege zur akademischen Qualifizierung im Sozial- und Gesundheitssystem. Berufsbegleitend studieren an Offenen Hochschulen. Münster: Waxmann.

Seger, M.S., Waldeyer, C., \& Leibinger, C. (2017). Qualitätssicherung im Kontext der Anrechnung und Anerkennung von Lernergebnissen an Hochschulen. Standards für zuverlässige, transparente und einheitliche Verfahren, Prozesse und Kriterien. Aachen: Shaker.

Sturm, N. (2016). Anerkennung und Anrechnung außerhochschulisch erworbener Kompetenzen. Ein Organisationsentwicklungsprojekt für deutsche Hochschulen. Dissertation, Philipps-Universität Marburg. Marburg/Lahn

Sturm (2018a). Anrechnungsberatung in der wissenschaftlichen Weiterbildung. Beratungswissen, Prozessstrukturen, Optimierungspotentiale. In W. Seitter, M. Friese \& P. Robinson (Hrsg.), Wissenschaftliche Weiterbildung zwischen Implementierung und Optimierung (S. 283-297). Wiesbaden: Springer VS.

Sturm, N. (2018b). Intraorganisationale Vorarbeiten als Voraussetzung für eine Implementierung von Anrechnungsverfahren. In A. Schulte, M. Wadewitz, M. Gercke, M. Gomille \& H. Schramm (Hrsg.), Vom Projekt zum Produkt - wissenschaftliche Weiterbildung für beruflich Qualifizierte an Hochschulen (S. 126-139). Detmold: Eusl.

Sturm, N., \& Hanak, H. (2018). Identifizierung der Kernprozesse in Anrechnungsverfahren: eine Voraussetzung für die nachhaltige Implementierung in Hochschulstrukturen? In T. C. Feld \& S. Lauber-Pohle (Hrsg.), Organisation und Profession. Felder erwachsenenpädagogischer Forschung (S. 181-194). Wiesbaden: Springer VS.

Voigt, H. (2012). Realisierungsbarrieren wissenschaftlicher Weiterbildung nach Bologna. Wissenschaftliche Weiterbildung der Hochschulreform der siebziger Jahre bis zum Bologna-Prozess. Hessische Blätter für Volksbildung. https://doi.org/10.3278/HBV1202W167. 